\title{
Imaging interstitial iron concentrations in boron-doped crystalline silicon using photoluminescence
}

\author{
D. Macdonald, ${ }^{1, \text { a) }}$ J. Tan, ${ }^{1}$ and T. Trupke ${ }^{2}$ \\ ${ }^{1}$ Department of Engineering, College of Engineering and Computer Science, \\ The Australian National University, Canberra, Australian Capital Territory 0200, Australia \\ ${ }^{2}$ Australian Research Council Centre of Excellence for Advanced Silicon Photovoltaics and Photonics, \\ University of New South Wales, Sydney 2052, Australia
}

(Received 27 November 2007; accepted 6 February 2008; published online 4 April 2008)

\begin{abstract}
Imaging the band-to-band photoluminescence of silicon wafers is known to provide rapid and high-resolution images of the carrier lifetime. Here, we show that such photoluminescence images, taken before and after dissociation of iron-boron pairs, allow an accurate image of the interstitial iron concentration across a boron-doped $p$-type silicon wafer to be generated. Such iron images can be obtained more rapidly than with existing point-by-point iron mapping techniques. However, because the technique is best used at moderate illumination intensities, it is important to adopt a generalized analysis that takes account of different injection levels across a wafer. The technique has been verified via measurement of a deliberately contaminated single-crystal silicon wafer with a range of known iron concentrations. It has also been applied to directionally solidified ingot-grown multicrystalline silicon wafers made for solar cell production, which contain a detectible amount of unwanted iron. The iron images on these wafers reveal internal gettering of iron to grain boundaries and dislocated regions during ingot growth. (C) 2008 American Institute of Physics.
\end{abstract}

[DOI: $10.1063 / 1.2903895]$

\section{INTRODUCTION}

Iron is a common and important impurity in solar-grade silicon materials such as ingot-grown multicrystalline silicon. ${ }^{1-4}$ The concentration of interstitial iron $\left[\mathrm{Fe}_{i}\right]$ can laterally vary across a wafer ${ }^{5}$ and along the length of an ingot ${ }^{4}$ and can also dramatically change during cell processing due to precipitate formation or dissolution and impurity gettering. ${ }^{2,6-11}$ It is therefore of considerable interest to be able to map changes in the iron concentration both laterally and during processing.

Several techniques have been developed over the years to perform this task, based on the measurement of carrier lifetimes, or diffusion lengths, before and after dissociation of FeB pairs. ${ }^{12}$ However, these techniques are relatively slow since they must measure these parameters point by point. The use of lifetime "imaging" methods rather than point-bypoint "mapping" methods would reduce the time required to obtain a high-resolution iron map from up to several hours to just minutes. This is in principle made possible by the recent advent of methods for rapid imaging of carrier lifetimes in silicon wafers, namely, infrared-lifetime mapping (ILM) ${ }^{13}$ also referred to as carrier density imaging, ${ }^{14}$ and photoluminescence (PL) imaging. ${ }^{15}$ In fact, Henze et al. ${ }^{16}$ recently used ILM to image interstitial iron concentrations in Ga-doped multicrystalline silicon wafers, revealing interesting effects near the grain boundaries.

In this paper, we introduce a method for such "iron imaging" using photoluminescence images taken before and after dissociation of FeB pairs. This approach offers the advantage that lifetime images can be obtained very rapidly (on the

${ }^{a)}$ Electronic mail: daniel.macdonald@anu.edu.au. order of one second) ${ }^{15}$ which reduces the amount of $\mathrm{FeB}$ pair breaking during the initial measurement (an important consideration for accurate iron imaging). Secondly, the PL images are immune to minority carrier trapping artifacts, ${ }^{17}$ which are prevalent in solar-grade silicon materials such as multicrystalline silicon. This means that the technique can be applied at low injection levels, further reducing the amount of pair breaking during the first measurement. Coupled with a generalized analysis that takes account of variations in injection level from pixel to pixel and within each pixel after pair breaking, this technique can produce accurate, fast, and high-resolution images of the interstitial iron concentration.

\section{EXISTING IRON MAPPING TECHNIQUES}

There are two main existing methods that can map the interstitial iron concentration in silicon wafers. The first, based on a method developed by Zoth and Bergholz, ${ }^{12}$ uses scanning surface-photovoltage (SPV) measurements of the low-injection carrier diffusion length. ${ }^{9,18,19}$ Later, based on the same principle, microwave-detected photoconductance decay ( $\mu \mathrm{W}$-PCD) maps of high-injection carrier lifetimes were used to generate iron maps, as shown for example, in Ref. 5. Both these approaches use point-by-point mapping and are hence quite slow. In addition, SPV is often limited to cases where the diffusion length is shorter than the wafer thickness (which would not lead to a very efficient solar cell). The $\mu \mathrm{W}-\mathrm{PCD}$ mapping technique is also subject to some uncertainty regarding variations in injection level, as discussed in detail below. Iron mapping based on infrared photothermal radiometry has also been recently developed. ${ }^{20}$

All of these techniques rely on the underlying principle initially developed by Zoth and Bergholz, ${ }^{12}$ who showed that 


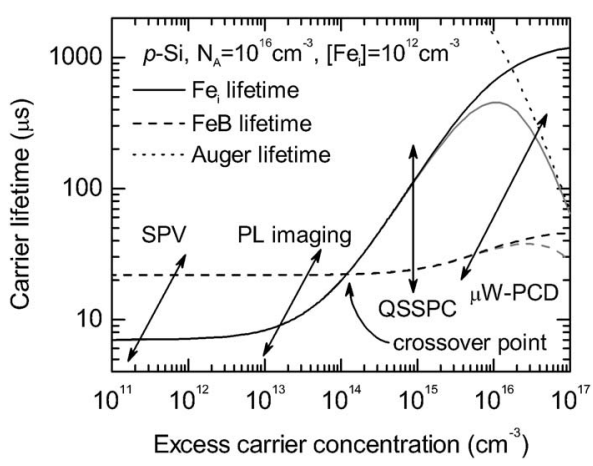

FIG. 1. The expected carrier lifetimes due to $\mathrm{Fe}_{i}$ and $\mathrm{FeB}$ pairs in borondope $p$-type silicon with $N_{A}=10^{16} \mathrm{~cm}^{-3}$ and an iron concentration of $10^{12} \mathrm{~cm}^{-3}$ as a function of the excess carrier concentration $\Delta n$. Auger recombination is also shown at high injection and its impact on the lifetimes due to $\mathrm{Fe}_{i}$ and $\mathrm{FeB}$. The injection regions in which the SPV, PL imaging, QSSPC, and $\mu \mathrm{W}-\mathrm{PCD}$ techniques typically operate are also indicated. Those shown with an angled arrow are constant generation rate techniques.

the interstitial iron concentration $\left[\mathrm{Fe}_{i}\right]$ can be found via measurement of the carrier lifetime (or, equivalently, the diffusion length), before and after breaking FeB pairs in a borondoped $p$-type silicon sample. The iron concentration is determined by ${ }^{10,12}$

$$
\left[\mathrm{Fe}_{i}\right]=C\left(\frac{1}{\tau_{1}}-\frac{1}{\tau_{0}}\right)
$$

where $\tau_{0}$ and $\tau_{1}$ are the lifetimes measured before and after breaking the FeB pairs. The conversion factor $C$ depends on the energy level and capture cross sections of the $\mathrm{Fe}_{i}$ and FeB states and the doping concentration. ${ }^{10}$

A critical consideration is that, in general, $C$ also depends on the injection level, or excess carrier density $\Delta n$, at which the two lifetime measurements are taken. ${ }^{10}$ Like many other techniques, SPV measurements are performed with the same generation rate for the two measurements before and after splitting the FeB pairs. This means that the injection level changes after splitting the FeB pairs, as indicated by the diagonal arrow labeled "SPV" in Fig. 1. However, in the case of the SPV technique, this does not complicate the determination of $C$. Because SPV operates under true lowinjection conditions, the lifetimes due to $\mathrm{Fe}_{i}$ and $\mathrm{FeB}$ pairs are injection independent, as can be seen in Fig. 1 at the lowest-injection levels. This results in a $C$ value that is also injection independent, significantly simplifying the application of the technique in the case of SPV measurements. In practice, the effective lifetime under low injection may still be affected by injection-dependent surface recombination. The low-injection levels used for SPV also help us to ensure that minimal FeB pair splitting occurs during the measurements themselves.

In contrast, techniques such as ILM and $\mu \mathrm{W}$-PCD must operate at higher injection levels since they are prone to carrier trapping and other trappinglike effects. ${ }^{17}$ In fact, they generally operate above the so-called "crossover point," shown in Fig. 1, and which occurs at an excess carrier density of around $1 \times 10^{14} \mathrm{~cm}^{-3}$ for $1 \Omega \mathrm{cm} \mathrm{B-doped} \mathrm{material.}{ }^{21}$ In the regime above the crossover point, the carrier lifetime increases after pair dissociation. Figure 1 indicates the region in which $\mu \mathrm{W}$-PCD measurements for Fe mapping are usually performed. It is clear that in this region, there is a strong injection dependence in the $\mathrm{Fe}_{i}$ lifetime and also due to the impact of Auger recombination. This means that, in principle, the conversion factor $C$ strongly depends on the local excess carrier density and therefore must be evaluated for each pixel since the injection level changes after pair dissociation and may vary from pixel to pixel.

Since PL imaging is immune to trappinglike artifacts, it can in principle be used in the true low-injection regime as for SPV. In practice though, PL imaging is better used at moderate injection levels, where the signal-to-noise ratio of the relatively weak luminescence is higher. In this work, we have taken PL images at excess carrier densities below the crossover point, as indicated again in Fig. 1. This was necessary because of the limited power of the laser diode array over a large area, which capped the available generation rate. However, the lower generation rate was also essential to avoid significant breaking of $\mathrm{FeB}$ pairs during the initial measurement. Note that the much higher-injection levels usually used for $\mu \mathrm{W}$-PCD measurements do not necessarily cause excessive pair breaking during measurement because the illumination is only present for a fraction of a second. By contrast, with PL or ILM imaging, which are steady-state techniques, the illumination must remain for seconds to minutes.

Nevertheless, at the moderate injection levels used for PL imaging, it is still necessary to account for the impact of changing injection levels on the $C$ factor. In this work, we therefore developed and applied a generalized method for calculating the local $\left[\mathrm{Fe}_{i}\right]$, pixel by pixel, taking these effects into account. It also accounts for the influence of Auger recombination and is therefore applicable to $\mu \mathrm{W}$-PCD iron mapping as well.

Note that an alternative to this approach is to simplify the determination of $C$ by ensuring that the two lifetime measurements are taken at the same value of $\Delta n$. This is illustrated in Fig. 1 by the vertical line labeled "QSSPC." This represents the excess carrier density at which quasi-steadystate photoconductance (QSSPC) lifetime measurements ${ }^{22}$ are usually taken at in order to determine area-averaged iron concentrations (over a few square centimeters, the size of the sense head on this apparatus). ${ }^{10}$ This is not generally possible with mapping or imaging methods.

Finally, it is important to note that, whichever technique is used to measure the lifetimes, it should be performed at carrier densities not too close to the crossover point since then the change in lifetime is very small and the technique is largely insensitive to iron. This may require different generation rates to be used for samples with very different lifetimes.

\section{A GENERALIZED APPROACH TO IRON MAPPING AND IMAGING}

Consider two steady-state lifetime measurements performed before and after FeB pair dissociation using the same generation rate $G$. During the initial measurement, the local excess carrier density $\Delta n_{0}$ is related to the initial lifetime $\tau_{0}$ 
via $\Delta n_{0}=G \tau_{0}$. Similarly, during the second measurement, $\Delta n_{1}=G \tau_{1}$. If we assume that all of the $\mathrm{Fe}_{i}$ is present as $\mathrm{FeB}$ pairs during the initial measurement and as isolated $\mathrm{Fe}_{i}$ during the final measurement, then the lifetimes can be written in terms of their components as

$$
\begin{aligned}
& \frac{1}{\tau_{0}}=\frac{1}{\tau_{\mathrm{FeB}}}+\frac{1}{\tau_{\text {Auger } 0}}+\frac{1}{\tau_{\text {other } 0}}, \\
& \frac{1}{\tau_{1}}=\frac{1}{\tau_{\mathrm{Fe}_{i}}}+\frac{1}{\tau_{\text {Auger } 1}}+\frac{1}{\tau_{\text {other } 1}},
\end{aligned}
$$

where $\tau_{\text {Auger0 }}$ and $\tau_{\text {Auger1 }}$ are the Auger lifetimes ${ }^{23}$ at $\Delta n_{0}$ and $\Delta n_{1}$, calculated according to the general parametrization in Ref. 23 , and $\tau_{\text {other } 0}$ and $\tau_{\text {other } 1}$ are the lifetimes due to all other recombination channels at $\Delta n_{0}$ and $\Delta n_{1}$. If we make the approximation that $\tau_{\text {other } 0} \approx \tau_{\text {other } 1}$ and subtract Eq. (1) from Eq. (2), the terms involving $\tau_{\text {other }}$ cancel out. We may then use the Shockley-Read-Hall expressions ${ }^{10,24,25}$ for $\tau_{\mathrm{Fe}_{i}}$ and $\tau_{\mathrm{Fe}_{B}}$, followed by some rearrangement and simplifications due to the specific values of the capture cross sections and energy levels, to find an expression for the local interstitial iron concentration $\left[\mathrm{Fe}_{i}\right]$,

$$
\left[\mathrm{Fe}_{i}\right]=\frac{1}{\chi^{\mathrm{Fe}_{i}}-\chi^{\mathrm{FeB}}}\left[\frac{1}{\tau_{1}}-\frac{1}{\tau_{0}}-\frac{1}{\tau_{\text {Auger } 1}}+\frac{1}{\tau_{\text {Auger } 0}}\right],
$$

where

$$
\begin{aligned}
& \chi^{\mathrm{Fe}_{i}}= \frac{v_{\mathrm{th}}\left(N_{A}+\Delta n_{1}\right)}{\frac{1}{\sigma_{n}^{\mathrm{Fe}_{i}}}\left(N_{A}+p_{1}^{\mathrm{Fe}_{i}}+\Delta n_{1}\right)+\frac{\Delta n_{1}}{\sigma_{p}^{\mathrm{Fe}_{i}}}}, \\
& \chi^{\mathrm{FeB}}=\frac{v_{\mathrm{th}}\left(N_{A}+\Delta n_{0}\right)}{\frac{1}{\sigma_{n}^{\mathrm{FeB}}}\left(N_{A}+\Delta n_{0}\right)+\frac{1}{\sigma_{p}^{\mathrm{FeB}}}\left(n_{1}^{\mathrm{FeB}}+\Delta n_{0}\right)},
\end{aligned}
$$

where $v_{\text {th }}$ is the thermal velocity, $\sigma$ is the capture cross sections (for electrons or holes and for $\mathrm{FeB}$ or $\mathrm{Fe}_{i}$ ), and $p_{1}$ and $n_{1}$ have their usual meanings in the Shockley-Read-Hall model. We have assumed a value of $1.1 \times 10^{7} \mathrm{~cm} / \mathrm{s}$ for $v_{\mathrm{th}}{ }^{26}$ The capture cross sections and energy levels for $\mathrm{FeB}$ and $\mathrm{Fe}_{i}$ are taken from Ref. 21, except for $\sigma_{n}^{\mathrm{Fe}_{i}}$ for which we have used a value of $1.3 \times 10^{-14} \mathrm{~cm}^{2}$. We have found that this value gives a better agreement with the implanted doses in the single-crystal float-zone (FZ) sample. Note that since we are operating below the crossover point in this case, our data are much more sensitive to this capture cross section than $\left[\mathrm{Fe}_{i}\right]$ measurements performed at higher injection, for example, with the QSSPC tool or with $\mu \mathrm{W}$-PCD. This is because the capture cross section for minority carriers (electrons in $p$-type silicon) dominates the carrier lifetime at lower injection levels for deep levels such as $\mathrm{Fe}_{i}$, whereas at higher injection levels, the smaller majority carrier capture cross section of $\mathrm{Fe}_{i}$ is dominant.

The parameters that are to be determined from the PL images then are $\Delta n_{0}$ and $\Delta n_{1}$ and $\tau_{0}$ and $\tau_{1}$. The method for converting the local PL count rates into local $\Delta n$ values is described in the following section. The $\tau$ values are then determined using $\Delta n=G \tau$, assuming that the excess carrier density profile is approximately uniform throughout the sample thickness at any given point on the wafer.

Using the expressions above to calculate the local $\left[\mathrm{Fe}_{i}\right]$ pixel by pixel accounts for the changing injection level and Auger recombination. These considerations are not generally taken into account in the application of $\mu \mathrm{W}$-PCD to produce iron maps. Indeed, it may be difficult to do so since, being a differential lifetime technique, the true value of $\Delta n$ is not always known. This would probably lead to some errors in Fe maps determined in this way, although the errors would hopefully be small.

Finally, it is worth noting that one of the most powerful aspects of the Zoth and Bergholz technique for determining iron concentrations is that the impact of other recombination channels, represented by $\tau_{\text {other }}$, is subtracted out when the two recombination rates (inverse lifetimes) are subtracted. This means that iron measurements can be taken even on samples that contain other significant sources of recombination. However, this approach is only valid if these other channels are not altered by the FeB pair breaking process. It is also only strictly valid if the two lifetimes $\tau_{0}$ and $\tau_{1}$ are measured at the same value of $\Delta n$ since only then can we be certain that the value of $\tau_{\text {other }}$ is the same in both cases. As discussed above, however, all mapping and imaging techniques necessarily use the same generation rate before and after pair splitting, meaning that the local $\Delta n$ value itself changes. Hence, there is no way to reliably subtract the impact of $\tau_{\text {other. }}$ This problem is, in principle, impossible to avoid for any method based on two lifetime measurements performed under the same generation rate, whether using $\mu \mathrm{W}$-PCD, ILM, or PL imaging. We can therefore only hope that such uncertainties remain small. This would certainly be true in the cases where the lifetime is dominated by iron, as is the case here, and when other recombination mechanisms and their injection dependence thus play a minor role. In cases where the injection dependence of $\tau_{\text {other }}$ is thought to distort the results, a comparison of two pairs of measurements with different generation rates could be used to reveal the presence of such an effect.

We firstly applied our generalized pixel-by-pixel analysis, coupled with PL imaging, to generate iron images of a single-crystal float-zone wafer with distinct regions of varied but known concentrations of implanted iron. This allowed us to verify that the technique generates reasonably accurate iron concentrations. We subsequently applied the method to "as-cut" multicrystalline silicon wafers from a directionally solidified ingot made for solar cell production. Such wafers contain a detectible amount of "natural" interstitial iron that arises from contamination from the crucible. ${ }^{2,4,27}$ The interstitial iron concentration in this material significantly varies across a wafer due to precipitation at internal crystal defects during ingot growth, thus providing an interesting application for the iron imaging technique.

\section{EXPERIMENTAL METHODS}

A deliberately iron-contaminated single-crystal silicon sample was prepared by ion implantation using $0.9 \Omega \mathrm{cm}$ boron-doped $p$-type float-zone silicon. After initial surface 
etching and cleaning, the sample was subjected to three separate implantations of $70 \mathrm{keV} \mathrm{Fe}$ ions. Each implantation covered an area of $30 \times 30 \mathrm{~mm}^{2}$, and the doses used were 1 $\times 10^{11}, 3 \times 10^{11}$, and $6 \times 10^{11} \mathrm{~cm}^{-2}$. The sample was lowered by $10 \mathrm{~mm}$ in between each implantation, causing the implanted regions to overlap, and resulting in five distinct regions with total $\mathrm{Fe}$ doses of $1 \times 10^{11}, 4 \times 10^{11}, 10 \times 10^{11}, 9$ $\times 10^{11}$, and $6 \times 10^{11} \mathrm{~cm}^{-2}$, each having a $10 \times 30 \mathrm{~mm}^{2}$ in size. After further surface cleaning, the sample was then annealed at $1000{ }^{\circ} \mathrm{C}$ for $40 \mathrm{~min}$ in oxygen, growing a thin oxide that acts as a surface passivating layer. The wafer had a thickness of $0.025 \mathrm{~cm}$, meaning that the implant doses correspond to $\mathrm{Fe}$ volume concentrations of $4 \times 10^{12}, 1.6 \times 10^{13}$, $4 \times 10^{13}, 3.6 \times 10^{13}$, and $2.4 \times 10^{13} \mathrm{~cm}^{-3}$. The solubility of Fe at the annealing temperature is about $4 \times 10^{14} \mathrm{~cm}^{-3}, 28$ which was intended to avoid precipitation of Fe during annealing. However, for safety reasons, the furnace was allowed to cool (over several minutes) to $900{ }^{\circ} \mathrm{C}$ before unloading, at which temperature the solubility limit is closer to $4 \times 10^{13} \mathrm{~cm}^{-3}$. It is therefore possible that some precipitation may have occurred in the regions with the highest doses. Note that the low ion energy and doses used here have previously been shown to leave negligible crystal damage after annealing at high temperature. ${ }^{29}$

The directionally solidified multicrystalline silicon (mc$\mathrm{Si}$ ) samples were standard, commercially grown $p$-type boron-doped $1.5 \Omega \mathrm{cm} \quad$ (approximately) wafers, 125 $\times 125 \mathrm{~mm}^{2}$ in size, and approximately $0.028 \mathrm{~cm}$ thick. After surface etching and cleaning, the samples were subjected to plasma-enhanced chemical vapor deposition of SiN films on both surfaces at $400{ }^{\circ} \mathrm{C}$ to provide surface passivation, while avoiding significant dissolution or precipitation of the naturally occurring iron in the wafers.

PL images were obtained with the system developed at UNSW, described in detail elsewhere. ${ }^{15}$ Steady-state carrier generation during imaging was achieved with an $815 \mathrm{~nm}$ laser diode array, the intensity of which could be varied. In this work, we used an incident photon flux of 2.5 $\times 10^{17} \mathrm{~cm}^{-2} \mathrm{~s}^{-1}$, corresponding approximately to 1 sun illumination strength. The band-to-band PL intensity radiating from the wafer was monitored by a one megapixel silicon charge-coupled device camera. PL images were collected for a duration of $1 \mathrm{~s}$. FeB pair dissociation was achieved by illuminating the wafers with the laser diode array for $1 \mathrm{~min}$ with 10 sun intensity before a second PL image was taken.

To create an iron image from the PL images, it is necessary to convert the measured PL count at each point into a local excess carrier density $\Delta n$. In this work, we achieved this by comparison to area-averaged carrier lifetime measurements performed with the QSSPC technique. ${ }^{22}$ Among other things, this method allows direct measurement of the average $\Delta n$ value under a given generation rate or photon flux. Comparison of this average $\Delta n$ value to the averaged PL count rate over the same region allows the linear conversion factor between the local PL count rate and the local $\Delta n$ value to be determined. For the conditions used here, this conversion factor was typically around $1 \times 10^{10} \mathrm{~cm}^{-3} /$ count. Note that our assumption that $\Delta n$ scales linearly with the PL intensity is valid if $\Delta n$ is significantly less than $N_{A}$ since in

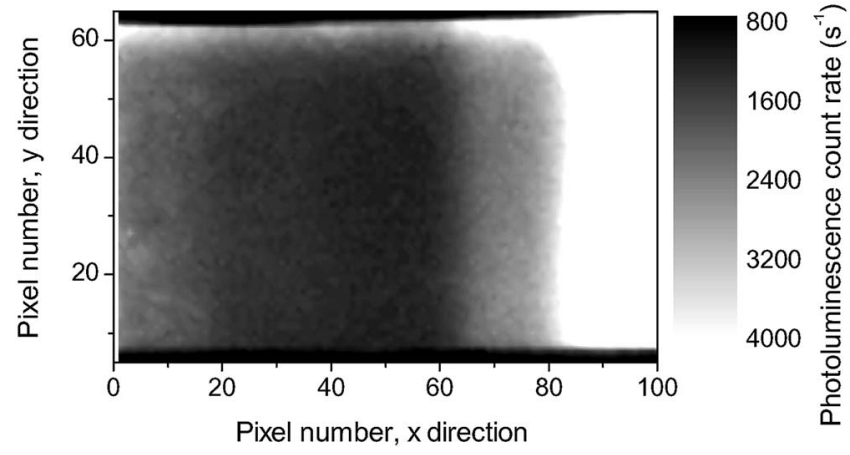

FIG. 2. Photoluminescence image of the Fe-implanted FZ sample before dissociation of the FeB pairs. The image was taken under a generation rate of 1 sun for a period of $1 \mathrm{~s}$, with the PL count rate shown on the right. The section of the sample shown was $5 \mathrm{~cm}$ wide and reveals the five regions of different implant doses.

general the PL intensity scales in proportion to $\Delta n\left(N_{A}+\Delta n\right) .{ }^{30}$ For the samples used in this work, $N_{A}$ $\sim 10^{16} \mathrm{~cm}^{-3}$ and the $\Delta n$ values are always below $10^{14} \mathrm{~cm}^{-3}$ (in order to remain below the crossover point, as described above). It is important to note that calibrating PL counts by comparison with QSSPC data can lead to systematic errors of up to $20 \%$ due to photon reabsorption. ${ }^{31,32}$ This would lead to a comparable uncertainty in the interstitial iron concentration, in addition to uncertainties from other sources.

\section{RESULTS AND DISCUSSION}

Figure 2 shows the initial PL image of the implanted FZ sample before $\mathrm{FeB}$ pair dissociation, taken for $1 \mathrm{~s}$ under a generation rate of 1 sun. The five regions of different $\mathrm{Fe}$ doses are evident, with the lightest dose on the right (highest lifetime and therefore highest PL count rate), and the heaviest Fe dose in the middle (lowest lifetime and PL count rate). The carrier lifetime in this sample varied from $1.1 \mu \mathrm{s}$ in the center to $4.9 \mu \mathrm{s}$ on the right, as determined from the PL intensities. Figure 3 shows a line scan of the PL count rate through this sample, from left to right, both before and after FeB pair dissociation. Note that the count rate has dramatically dropped after pair dissociation, indicating that the lifetime has also dropped, to $0.3 \mu \mathrm{s}$ in the center and $1.6 \mu \mathrm{s}$ on the right. This is consistent with the fact that the excess carrier densities were in the range of $2 \times 10^{12}-5$ $\times 10^{13} \mathrm{~cm}^{-3}$, well below the crossover point.

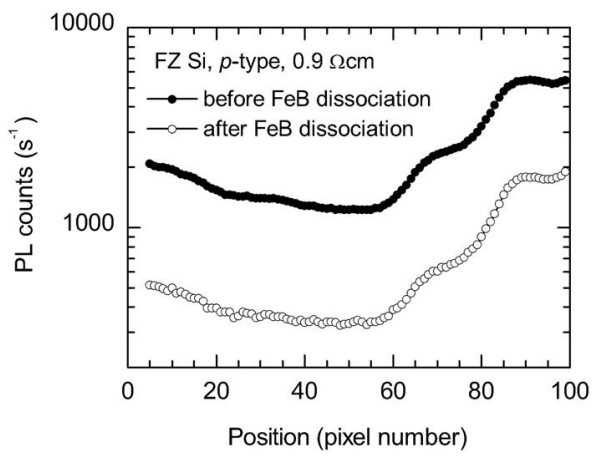

FIG. 3. Line scan of the PL intensity of the FZ sample of Fig. 2, from left to right, before and after FeB pair dissociation. 


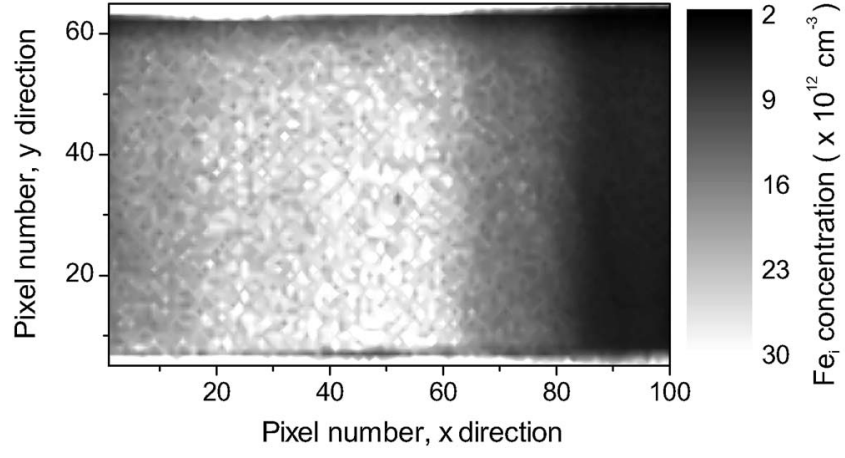

FIG. 4. Calculated iron image of the FZ sample in Fig. 2. The concentration of $\mathrm{Fe}_{i}$ is shown on the right in units of $\mathrm{cm}^{-3}$.

By contrast, higher generation rates resulting in $\Delta n$ values above the crossover point caused an increase in the PL intensity after $\mathrm{FeB}$ pair dissociation, as expected. However, it proved difficult to avoid significant dissociation of FeB pairs during the first measurement when using illumination intensities significantly greater than 1 sun for this sample. This can dramatically reduce the accuracy of the technique. Although the additional pair breaking can be partly offset by reducing the data collection time, unfortunately the FeB dissociation rate increases approximately quadratically with intensity. ${ }^{33}$ It is therefore more accurate to perform iron imaging with PL at $\Delta n$ values below the crossover point, as we have done here. This also suggests that to improve the signal to noise ratio in the luminescence detection, it is advisable to use longer exposure times with low light intensity since the count rate is linear with exposure time, rather than opting for higher illumination intensities.

Figure 4 shows the PL data converted into an iron image using the expressions presented above. The $\left[\mathrm{Fe}_{i}\right]$ data are noisier than the raw PL data in Fig. 2 mainly because the second PL image, taken after pair dissociation, was noisier than the first (because of the reduced lifetime). Nevertheless, the regions of different $\mathrm{Fe}$ concentration are visible, although there is some blurring between the regions due to lateral diffusion of Fe during the annealing. The Fe concentrations obtained in each region were averaged (only taking data from the center of each region where the Fe concentrations were nearly constant) and are plotted again in Fig. 5 versus the $\mathrm{Fe}$ concentration expected from the implantation dose (solid symbols). In general, the measurements are in reasonable agreement with the expected values, although there appears to be a slight systematic underestimation of $\left[\mathrm{Fe}_{i}\right]$ for the two highest dosees. This is likely due to precipitation of some $\mathrm{Fe}_{i}$ during cooling after annealing, as discussed above.

Also shown in Fig. 5 is the data from multicrystalline silicon wafers containing natural iron (open symbols). For these wafers, we have averaged the interstitial iron concentration from the PL iron image over a region of several $\mathrm{cm}^{2}$. This has then been plotted against the iron concentration measured over the same region by a pair of area-averaged QSSPC lifetime measurements taken before and after pair dissociation. ${ }^{10,34}$ The QSSPC $\left[\mathrm{Fe}_{i}\right]$ values were determined at an excess carrier density of $1 \times 10^{15} \mathrm{~cm}^{-3}$, as is typical for this technique. Although this is above the crossover point, as

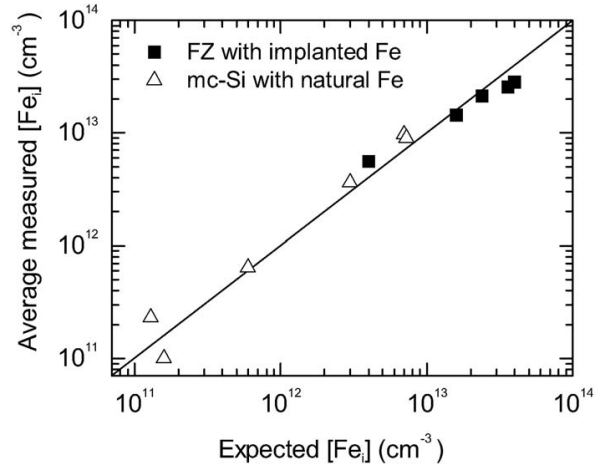

FIG. 5. Average measured interstitial iron concentration $\left[\mathrm{Fe}_{i}\right]$ obtained from PL iron images, plotted against the expected value of $\left[\mathrm{Fe}_{i}\right]$. The solid line represents exact agreement between the measurements. The solid symbols indicate the five regions of the $\mathrm{FZ}$ sample, where the expected $\left[\mathrm{Fe}_{i}\right]$ values are directly calculated from the implanted Fe dose. The open symbols indicate multicrystalline silicon samples containing natural iron, for which the expected $\left[\mathrm{Fe}_{i}\right]$ values were independently measured using area-averaged QSSPC lifetime measurements.

opposed to the PL images, the agreement between the two techniques is encouraging.

Figure 6 shows the initial PL image of one of these mc-Si wafers before pair dissociation. This wafer was from approximately $20 \%$ above the bottom of the ingot, i.e., a region which usually contains significant quantities of iron. The PL data indicates that there are narrow regions near the grain boundaries that actually have higher lifetimes than deeper within the grains. However, the grain boundaries themselves have a lower lifetime, as indicated by the thin dark lines within the brighter regions. These observations indicate that internal gettering of impurities to the grain boundaries has occurred during ingot cooling, as has often been observed in this type of material. ${ }^{35-39}$

Figure 7 shows the corresponding Fe image generated from the pair of PL images. This shows that the interstitial iron concentration is dramatically reduced near the grain boundaries and highly dislocated areas, confirming that interstitial iron is indeed one of the impurities that are inter-

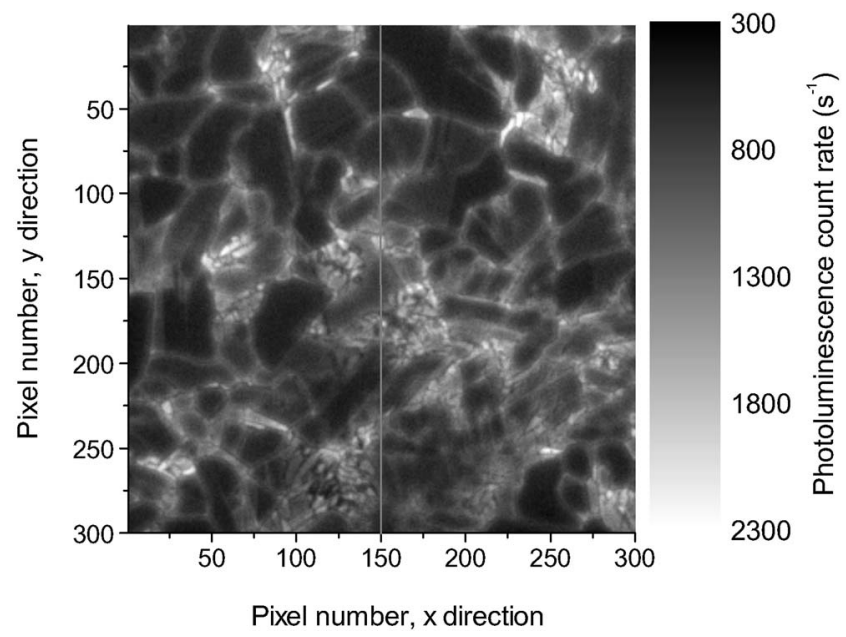

FIG. 6. Photoluminescence image of a multicrystalline silicon sample before dissociation of $\mathrm{FeB}$ pairs. The image was taken under a generation rate of 1 sun for a period of $1 \mathrm{~s}$, with the PL count rate shown on the right. The section of the sample shown was $5 \mathrm{~cm}$ wide. 


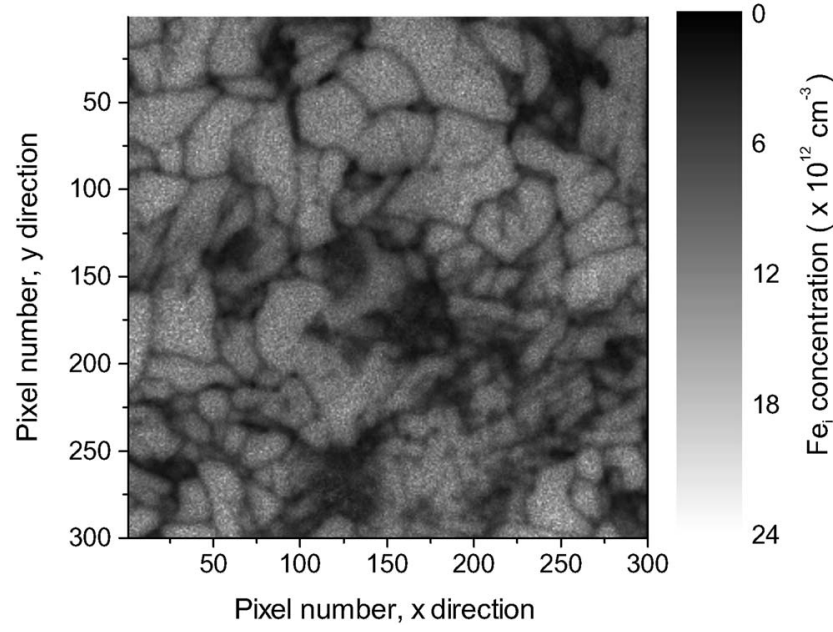

FIG. 7. Calculated iron image of the multicrystalline silicon sample in Fig. 6. The concentration of $\mathrm{Fe}_{i}$ is shown on the right in units of $\mathrm{cm}^{-3}$.

nally gettered. In the other parts of this wafer, the interstitial Fe concentration is surprisingly uniform from grain to grain. This may reflect the fact that these wafers are supersaturated with Fe during ingot cooling, ${ }^{4}$ and that the final intragrain $\mathrm{Fe}_{i}$ concentration is driven largely by the solid solubility limit and precipitation energetics, which are common from grain to grain. The average value of $\left[\mathrm{Fe}_{i}\right]$ in the iron image of Fig. 7 is $9 \times 10^{12} \mathrm{~cm}^{-3}$. This compares well with the average $\left[\mathrm{Fe}_{i}\right]$ measured on this wafer using the QSSPC technique which was $7 \times 10^{12} \mathrm{~cm}^{-3}$.

Figure 8 shows a line scan of the PL count rate along the vertical line shown in Fig. 6, both before and after FeB pair dissociation, in analogy to Fig. 3 for the FZ sample. Again, it is evident that the images were taken at excess carrier densities well below the crossover point. Note that the PL intensity is greatest and changes the least upon dissociation, near the grain boundaries, reflecting the lower $\mathrm{Fe}_{i}$ concentrations there.

Finally, it is striking to notice the strong anticorrelation between the two images in Figs. 6 and 7-regions of low lifetime correspond to regions of high $\left[\mathrm{Fe}_{i}\right]$. This would suggest that $\mathrm{Fe}_{i}$ has a strong impact on the carrier lifetime in wafers from this region of a mc-Si ingot. Indeed, we know this to be true from other measurements of $\left[\mathrm{Fe}_{i}\right]$ based on QSSPC lifetime measurements. ${ }^{34}$

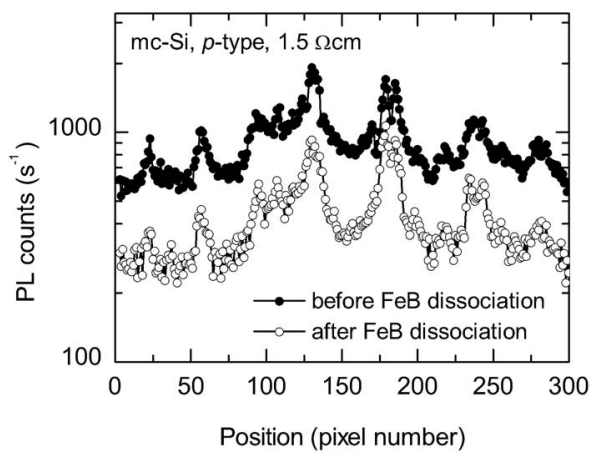

FIG. 8. Line scan of the PL intensity of the multicrystalline silicon sample along the vertical line at pixel number 150, shown in Fig. 6, from top to bottom, before and after FeB pair dissociation.

\section{CONCLUSIONS}

A technique for imaging the interstitial iron concentration across $p$-type silicon wafers based on band-to-band photoluminescence imaging has been developed. The method is rapid and offers high spatial resolution. It is suited to studying the behavior of iron, and by analogy other metal impurities, in photovoltaic silicon materials such as multicrystalline and ribbon silicon during ingot growth and cell processing. With the advent of less pure, solar-grade, silicon feedstock materials, such techniques will become increasingly useful. An important feature of PL imaging for the generation of iron images is the speed with which the PL images can be generated ( $1 \mathrm{~s}$ under 1 sun was used here). This enables the initial measurement to be carried out with minimal breaking of $\mathrm{FeB}$ pairs. While applied to iron in boron-doped silicon here, the technique could in principle be extended to other metal-acceptor pairs in silicon, such as $\mathrm{CrB}, \mathrm{MnB}$, and $\mathrm{FeGa}$, for example.

\section{ACKNOWLEDGMENTS}

D.M. is supported by an Australian Research Council QEII Fellowship. The Centre of Excellence for Advanced Silicon Photovoltaics and Photonics at UNSW is funded by the Australian Research Council. The authors are grateful to R. Elliman of the Research School of Physical Science and Engineering, ANU, for access to the ion implanter, to Eugenia Sepulveda for assistance with sample preparation, and to R. Bardos and M. Abbott at UNSW for assistance with PL imaging.

${ }^{1}$ A. A. Istratov, T. Buonassisi, R. J. McDonald, A. R. Smith, R. Schindler, J. A. Rand, J. P. Kalejs, and E. R. Weber, J. Appl. Phys. 94, 6552 (2003).

${ }^{2}$ T. Buonassisi, A. A. Istratov, M. Heuer, M. A. Marcus, R. Jonczyk, J. Isenberg, B. Lai, Z. Cai, S. Heald, W. Warta, R. Schindler, G. Willeke, and E. R. Weber, J. Appl. Phys. 97, 074901 (2005).

${ }^{3}$ A. Bentzen, A. Holt, R. Kopecek, G. Stokkan, J. S. Christensen, and B. G. Svensson, J. Appl. Phys. 99, 093509 (2006).

${ }^{4}$ D. Macdonald, A. Cuevas, A. Kinomura, Y. Nakano, and L. J. Geerligs, J. Appl. Phys. 97, 033523 (2005).

${ }^{5}$ O. Palais, S. Martinuzzi, and J. J. Simon, Mater. Sci. Semicond. Process. 4, 27 (2001).

${ }^{6}$ S. A. McHugo, Appl. Phys. Lett. 71, 1984 (1997).

${ }^{7}$ P. Zhang, H. Väinölä, A. A. Istratov, and E. R. Weber, Appl. Phys. Lett. 83, 4324 (2003)

${ }^{8}$ T. Buonassisi, A. A. Istratov, S. Peters, C. Ballif, J. Isenberg, S. Riepe, W. Warta, R. Schindler, G. Willeke, Z. Cai, B. Lai, and E. R. Weber, Appl. Phys. Lett. 87, 121918 (2005).

${ }^{9}$ L. Jastrzebski, W. Henley, D. Schielein, and J. Lagowski, J. Electrochem. Soc. 142, 3869 (1995).

${ }^{10}$ D. Macdonald, L. J. Geerligs, and A. Azzizi, J. Appl. Phys. 95, 1021 (2004).

${ }^{11}$ S. Dubois, O. Palais, P. J. Ribeyron, N. Enjalbert, M. Pasquinelli, and S. Martinuzzi, J. Appl. Phys. 102, 083525 (2007).

${ }^{12}$ G. Zoth and W. Bergholz, J. Appl. Phys. 67, 6764 (1990).

${ }^{13}$ M. Bail, J. Kentsch, R. Brendel, and M. Schulz, Proceedings 28th IEEE Photovoltaic Specialists Conference, Anchorage, Alaska (IEEE, New York, 2000), p. 99.

${ }^{14}$ J. Isenberg, S. Riepe, S. W. Glunz, and W. Warta, J. Appl. Phys. 93, 4268 (2003).

${ }^{15}$ T. Trupke, R. A. Bardos, M. C. Schubert, and W. Warta, Appl. Phys. Lett. 89, 044107 (2006).

${ }^{16}$ J. Henze, P. Pohl, C. Schmiga, M. Dhamrin, T. Saitoh, I. Yamaga, and J. Schmidt, Proceedings 20th European Photovoltaic Solar Energy Conference, Barcelona (WIP-Munich, Germany, 2005), p. 769.

${ }^{17}$ R. A. Bardos, T. Trupke, M. C. Schubert, and T. Roth, Appl. Phys. Lett. 
88, 053504 (2006)

${ }^{18}$ J. Lagowski, P. Edelman, M. Dexter, and W. Henley, Semicond. Sci. Technol. 7, A185 (1992).

${ }^{19}$ D. K. Schroder, Meas. Sci. Technol. 12, R16 (2001).

${ }^{20}$ M. E. Rodriguez, A. Mandelis, G. Pan, J. A. Garcia, V. Gorodokin, and Y. Raskin, J. Appl. Phys. 87, 8113 (2000).

${ }^{21}$ D. Macdonald, T. Roth, P. N. K. Deenapanray, T. Trupke, and R. A. Bardos, Appl. Phys. Lett. 89, 142107 (2006).

${ }^{22}$ R. A. Sinton and A. Cuevas, Appl. Phys. Lett. 69, 2510 (1996).

${ }^{23}$ M. J. Kerr and A. Cuevas, J. Appl. Phys. 91, 2473 (2002).

${ }^{24}$ W. Shockley and W. T. Read, Phys. Rev. 87, 835 (1952).

${ }^{25}$ R. N. Hall, Phys. Rev. 87, 387 (1952).

${ }^{26}$ W. M. Bullis and H. R. Huff, J. Electrochem. Soc. 143, 1399 (1996).

${ }^{27}$ E. Olsen and E. J. Øvrelid, Prog. Photovoltaics 16(2), 93 (2008).

${ }^{28}$ A. A. Istratov, H. Hieslmair, and E. R. Weber, Appl. Phys. A: Mater. Sci. Process. 69, 13 (1999).

${ }^{29}$ D. Macdonald, P. N. K. Deenapanray, and S. Diez, J. Appl. Phys. 96, 3687 (2004).

${ }^{30}$ T. Trupke, R. A. Bardos, and M. D. Abbott, Appl. Phys. Lett. 87, 184102
(2005)

${ }^{31}$ T. Trupke, J. Appl. Phys. 100, 063531 (2006).

${ }^{32}$ M. Rüdiger, T. Trupke, P. Würfel, R. Bardos, and T. Roth, Proceedings 22nd European Photovoltaic Solar Energy Conference, Milan, Italy (WIPRenewable Energies, Germany, 2007), p. 386.

${ }^{33}$ L. J. Geerligs and D. Macdonald, Appl. Phys. Lett. 85, 5227 (2004).

${ }^{34}$ J. Tan, D. Macdonald, N. Bennet, D. Kong, A. Cuevas, and I. Romijn, Appl. Phys. Lett. 91, 043505 (2007).

${ }^{35}$ L. L. Kazmerski, P. J. Ireland, and T. F. Ciszek, Appl. Phys. Lett. 36, 323 (1980).

${ }^{36}$ M. Kittler, W. Seifert, M. Stemmer, and J. Palm, J. Appl. Phys. 77, 3725 (1995).

${ }^{37}$ J. Lu, M. Wagener, G. Rozgonyi, J. Rand, and R. Jonczyk, J. Appl. Phys. 94, 140 (2003).

${ }^{38}$ J. Chen, T. Sekiguchi, D. Yang, F. Yin, K. Kido, and S. Tsurekawa, J. Appl. Phys. 96, 5490 (2004).

${ }^{39}$ T. Buonassisi, A. A. Istratov, M. D. Pickett, M. A. Marcus, T. F. Ciszek, and E. R. Weber, Appl. Phys. Lett. 89, 042102 (2006). 